\title{
Public perception of ethical issues concerning automated mobility
}

\author{
A focus group study among three road user categories
}

\author{
Ebru Dogan* \\ VEDECOM Institute, Versailles, \\ France \\ ebru.dogan@vedecom.fr
}

\author{
Cécile Barbier \\ University of Geneva, Geneva, \\ Switzerland \\ cecile.barbier@unige.ch
}

\author{
Estelle Peyrard \\ Institut Interdisciplinaire de \\ l'Innovation (UMR 9217), CRG, Ecole \\ Polytechnique, Palaiseau, France \\ estelle.peyrard@apf.asso.fr
}

\begin{abstract}
As the deployment of automated vehicles (AV) progresses, questions about the public acceptance and ethical issues raised by automated mobility emerge. The present focus group study aimed at examining the ethical issues related to the AVs as perceived by road users through two scenarios. Nineteen participants in three groups, namely, drivers, pedestrians, and road users with disabilities, participated in the study. The three major principles that concerned all road user groups were responsibility, human autonomy, and wellbeing. For the road users with disabilities social justice was additionally a paramount issue, while for drivers it was privacy. The scenarios revealed both similarities and discrepancies in worries and expectations of different road user categories.
\end{abstract}

\section{CCS CONCEPTS}

- General and reference $\rightarrow$ Document types; General conference proceedings conference proceedings; • Human-centered computing $\rightarrow$ Human computer interaction (HCI); HCI design and evaluation methods; User studies.

\section{KEYWORDS}

Ethics, Automated vehicle, Transport, Public Acceptance, Vulnerable road users

ACM Reference Format:

Ebru Dogan*, Cécile Barbier, and Estelle Peyrard. 2021. Public perception of ethical issues concerning automated mobility: A focus group study among three road user categories. In European Conference on Cognitive Ergonomics 2021 (ECCE 2021), April 26-29, 2021, Siena, Italy. ACM, New York, NY, USA, 6 pages. https://doi.org/10.1145/3452853.3452877

The deployment of automated vehicles (AV) premises changes in individual and public transport, while raising questions about public acceptance, including ethical issues. The critical situations that require ethical considerations were quickly compared to the "trolley problem" for application of different ethical frameworks [1-5]. This research shows a social dilemma in people's approach to AV. That is, people have conflicting opinions when they think of their interests

Permission to make digital or hard copies of part or all of this work for personal or classroom use is granted without fee provided that copies are not made or distributed for profit or commercial advantage and that copies bear this notice and the full citation on the first page. Copyrights for third-party components of this work must be honored

For all other uses, contact the owner/author(s).

ECCE 2021, April 26-29, 2021, Siena, Italy

(C) 2021 Copyright held by the owner/author(s).

ACM ISBN 978-1-4503-8757-6/21/04.

https://doi.org/10.1145/3452853.3452877 versus the greater good of society, which paves the way to the ethical challenges at a societal level. While the focus has always been on the behavior of a single AV in a critical situation, scaling up the behavior of the AV to the entire transport system is likely to have consequences for society, beyond the question of which moral code to implement in the AV [6].

Laypeople rely on their intuitions and socio-cognitive constructs, such as trust, value congruency, emotions, and contextual factors in their judgments of moral acceptability of new, complex technology, instead of crunching numbers like experts do [7]. In this respect, people become increasingly concerned about the vulnerability of artificial intelligence technologies to privacy breaches, data protection, consent, and security in general. AV is not an exception to this skepticism. There is a rising public interest in the implications of AV in terms of accessibility and fairness. Nonetheless, a comprehensive analysis showed that the research on the non-tangible implications of automated transportation, such as the implications for social equity and public health, are relatively under-researched [8]. Additionally, while the driver needs and use have been heavily studied, vulnerable road user categories, such as pedestrians, elderly road users, road users with disabilities, children, were overlooked.

The current manuscript reports the results of a focus groups study that was carried out as part of the H2020 project SUaaVE (Supporting acceptance of automated vehicles, 2019-2022) in order to comprehend public perception on the ethical issues raised by $\mathrm{AV}$, targeting different road user groups, namely, drivers, pedestrians, and road users with disabilities (RUD).

\section{METHOD}

\subsection{Participants}

Nineteen people (6 males) with the mean age of 39 -years $(\mathrm{SD}=6.5)$ participated in the current study, divided into three groups, namely, drivers (6 people), pedestrians (7 people), and RUD (6 people).

\subsection{Scenarios}

We defined three scenarios related to automated urban mobility for the focus group discussions. Transgression of a traffic rule depicted an $\mathrm{AV}$ that has to decide whether or not to comply with a prohibited action (i.e. crossing a continuous lane line) in order to overtake a stranded vehicle blocking the traffic circulation (Figure 1, left). Changes in urban landscape depicted potential changes in the use of (shared) space, provision of public and private transport services, 

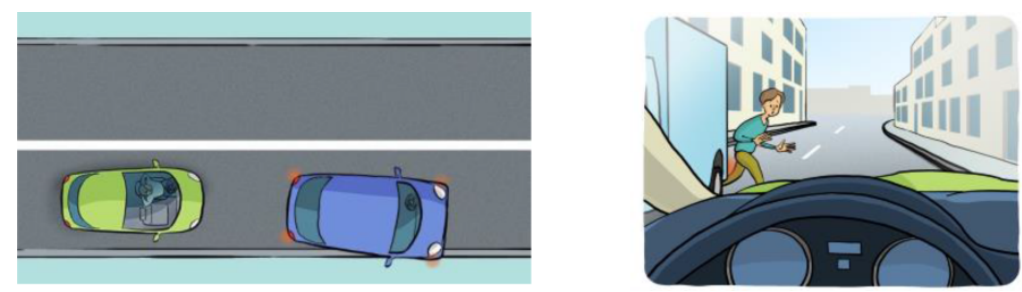

Figure 1: Illustration of the scenario of transgression of a traffic rule (left) and unavoidable accident (right)

commuting habits, and air pollution that might occur with the deployment of $\mathrm{AV}$ in the urban transport system. Unavoidable accident depicted an imminent impact between the $\mathrm{AV}$ and a pedestrian that was initially invisible to the sensors of the vehicle because he was behind a delivery truck (Figure 1, right).

\subsection{Ethical principles}

Six ethical principles had been identified in a previous focus group with experts in philosophy, robotics, urban design, human factors, and legal issues (December 2019): responsibility, autonomy, wellbeing, democracy, justice, and privacy/ data protection.

\subsection{Procedure}

A focus group methodology, supplemented with individual written phases, was adopted. The aim of the individual phases was to collect participants' opinion without the group effect and to anchor their individual point of view to launch the discussions. The aims of the group discussions were to create a (controversial) debate and observe the social influence on the evolution of opinions.

After the introduction and signing of the consent forms, two video clips explaining the AV technology were used as an icebreaker. In Step 1, not reported here, participants expressed their general take on the AV on sticky notes, followed by $10 \mathrm{~min}$ of group discussion. In Step 2, we presented participants with the six ethical principles from the expert focus group and defined each of them Participants, then, wrote on a sticky note the three principles that they considered indispensable to take into account for the deployment of AV, followed by a 25-30 min discussion. In Step 3 on scenarios, participants expressed on sticky notes which ethical issues they considered relevant for the scenario, followed by a group discussion of $50 \mathrm{~min}$ in total for three scenarios. Each session lasted 2 hours and the study was conducted the last week of February 2020.

\section{RESULTS}

The current manuscript is focused on results of Step 2 and two scenarios in Step 3. We will provide verbatims in italics to exemplify the expressions and opinions of participants.

\subsection{Step 2: selection of ethical principles}

The analysis of the three ethical principles considered absolutely necessary is presented in Table 1 and the mapping of responses are presented in Figure 2.

Responsibility - Who would be held responsible in case of an accident is identified as the most relevant ethical issue by all groups. Participants recognized that there were a lot of stakeholders involved in the AV ecosystem and expressed concerns about the discharge of responsibility by the industrial stakeholders, so that in the end the responsibility falls on the user, as in the case of "the problem of many hands" [9]. "There is no zero accident. So, it boils down to responsibility". "There are too many actors, too many responsibilities."

Drivers group distinguished legal responsibility and moral responsibility, stating in consensus that they would morally feel responsible for killing or injuring someone, even if they would not be held responsible legally. "If we kill someone, we will have to live with that feeling. It is not only a matter of jail or insurance money."

The next prominent ethical principle was human autonomy among all groups. The impact of the deployment of AV on autonomy was perceived rather negatively. Drivers mainly emphasized remaining, delegating control, out of the loop, and the diminished of capacity to react after a period of automated driving. Additionally, becoming dependent on technology was a major concern among drivers and pedestrians. "It becomes like mobile phone. I don't take initiative while using phone. What if I start leaving every decision to my car?" "Today we can deactivate [the automated mode], but if tomorrow they produce only fully automated vehicle, what do we do?"

On the positive side, participants recognized that the AV could improve the autonomy of currently immobile road users. In the RUD group, AV is sometimes considered enabling freedom. "For healthy people, the primary concern is "I will lose my autonomy and control". However, for some it may be a source of autonomy to get out of isolation." "I said autonomy because. . I will have more freedom."

Wellbeing was ranked third and expressed on various dimensions, such as gaining time, being able to engage in other activities, comfort and less fatigue during the ride, improved road safety, and environmental wellbeing (regarding pollution and how the battery of the AV is handled), common among all three groups. Nonetheless, pedestrians had some safety concerns about the AV's interactions with pedestrians, such as whether they will be able to understand that the AV has detected them or to anticipate the vehicle's behavior. "Crossing the street may be more stressful due to lack of anticipation [of the AV behavior]". "Since AV is a robot, does it mean that the pedestrian will always be at fault in case of an accident?". Among the RUD, wellbeing, like autonomy, was associated with mobility gains.

Privacy was expressed heavily by the drivers. Drivers stated they had the feeling of being followed and detected all the time even with the current technologies, a situation likely to aggravate with $\mathrm{AV}$, and argued the necessity of the possibility of deleting one's 
Table 1: The analysis of the top 3 ethical principles partitioned among the three groups

\begin{tabular}{lllllll}
\hline & Wellbeing & Autonomy & Privacy & Justice & Democracy & Responsibility \\
\hline Drivers & 3 & 5 & 4 & - & - & 6 \\
Pedestrians & 2 & 4 & 1 & 1 & 2 & 5 \\
RUD & 3 & 5 & - & 5 & 1 & 5 \\
\hline
\end{tabular}

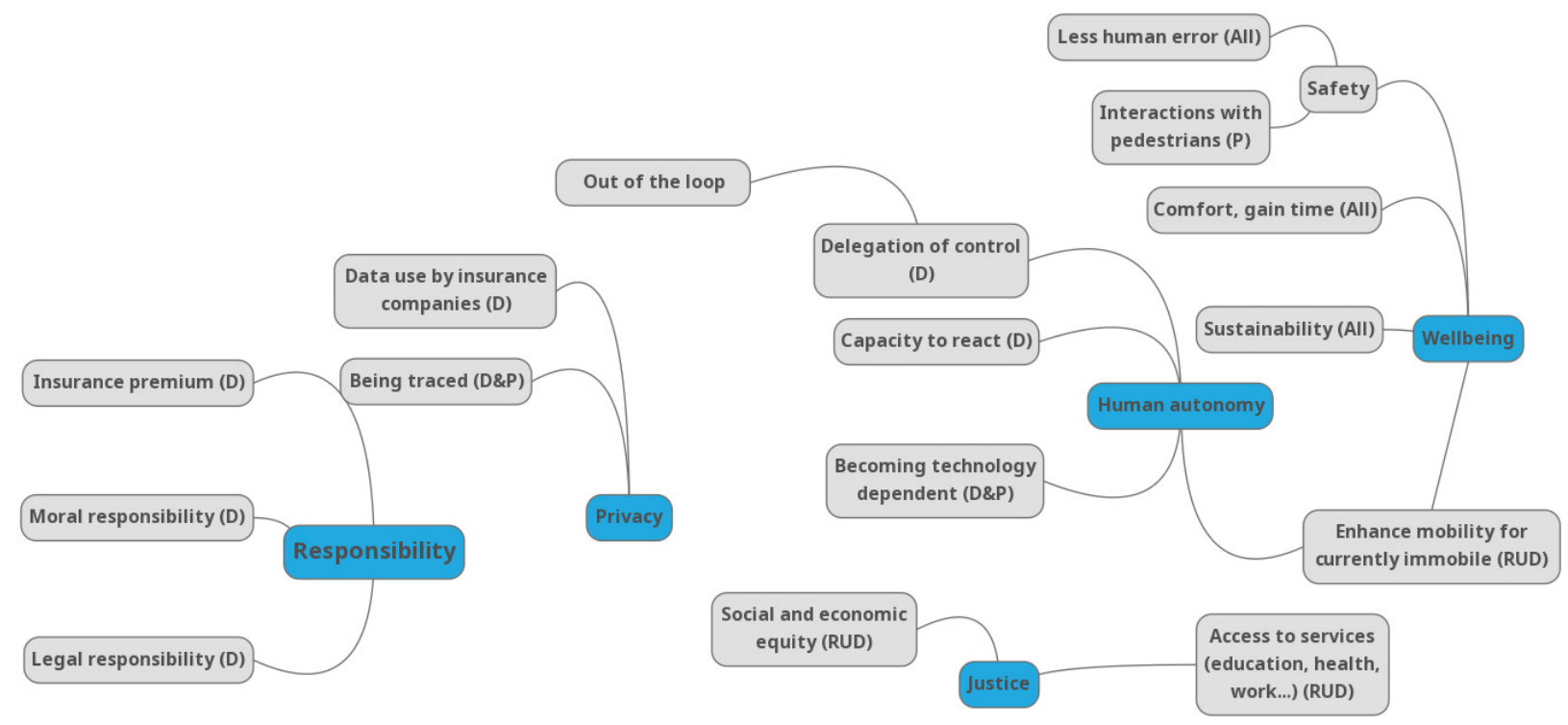

Figure 2: Mapping responses in Step 2

data. Data protection and financial concerns seem to interact with concerns that the insurance companies would use the personal data obtained from the AV to calibrate insurance premium. The RUD group was divided on the question of privacy. For half of the group, holding driver's license, it was a major concern. "Privacy comes first. The fact that we are tracked, that they know everything, were we go, with whom... We are not free." "Where do the data go? To the car manufacturer?" For the other half, not holding driver's license, on the contrary, privacy was not a major issue.

Social justice was predominantly prioritized by the RUD group and not mentioned at all by the drivers. For the RUD, social justice seems to have two dimensions. The first one is the role of AV in the improvement of mobility, for instance in access to work and education, since disabilities often impede the use of personal car or the access to public transport. The other dimension is the social and economic inequalities to purchase AV. Vehicles with specifications for RUD are already more expensive than regular vehicles. Hence, for the RUD group AV could exacerbate already existing social and economic inequalities. There was a consensus that the deployment of AV especially in public transport should bear in mind inclusiveness to improve the condition of RUD. "AV is supposed to contribute to social equity to improve the conditions of everybody, not only one [privileged] part of the society. We can imagine AV in public transport, in addition to personal use."
Although democracy was mentioned by three people in total, it did not come up during the discussions.

\subsection{Step 3: Scenarios}

2.2.1 Scenario 1: Transgression of a traffic rule. The main inquiries of this scenario were what participants thought about permitting an AV to transgress a traffic rule and whether it would be acceptable to design a machine that could violate the law. We grouped the answers written on the sticky notes in Table 2.

The striking difference among the three groups at first glance is the drivers' higher tolerance for the transgression of the traffic rules in contrast to the pedestrian and RUD groups' insistence on the impermissibility of a transgression. However, drivers insisted that this should be exceptional, for justified reasons, and still within the regulatory framework. Hence, paradoxically, drivers would want the violation of law to be defined within the law to avoid infringement of liability.

When the possibility of letting the AV learn these exceptional situations via machine learning algorithms instead of regulatory bodies defining them individually was raised, one participant said "I want the vehicle still to be a vehicle, not a person. A vehicle does not think!". Thus, we see a worry about anthropomorphism attributed to AV. Two other participants, also in the driver group, expressed 
Table 2: Opinions on the transgression scenario across three groups

\begin{tabular}{|c|c|c|c|}
\hline & Drivers & Pedestrians & RUD \\
\hline No violation of a traffic rule should be permitted. & 1 & 4 & 3 \\
\hline Yes, it can violate a traffic rule exceptionally/ out of necessity/ to avoid blocking the circulation. & 3 & 1 & 2 \\
\hline Yes, on the condition that the maneuver is carried out safely. & 2 & 1 & 1 \\
\hline A human should carry out the maneuver/ to decide on vehicle's behavior. & 1 & 2 & - \\
\hline
\end{tabular}

skepticism about the "cognitive capacities [of the AI applications in general] given the complexity of real-life situations".

Few participants in drivers and pedestrians groups proposed that a human decides vehicle's behavior. The possibilities of asking the driver or a remote operator to take over control of the AV were evoked.

One participant in the RUD group rejected transgression of traffic rules because it may result in people behaving irresponsibly, for instance, consuming more alcohol than the legal limit knowing that the AV would be able to handle any situation on the road. The AV could be considered to leave room for complacency.

Two common concerns raised in all groups were the extent to which we can keep defining exceptional cases and the impact on the non-automated mobility as a result of adopting the traffic rules to accommodate AV.

2.2.2 Scenario 2: Urban mobility. The interest of this scenario were how people imagined future cities with $\mathrm{AV}$ and what kind of compromises were considered acceptable. As in the first scenario, we grouped participants' responses in Table 3.

The most prominent opinion expressed among all user groups was that the AV should be riding on a dedicated lane segregated from the rest of the road users. In the pedestrian group, this was practically the only response. The main motivation behind this suggestion was concerns for safety and efficiency (e.g. the AV being blocked due to uncourteous behaviors of pedestrians or non-AVs). In a previous focus group with experts, an expert on urban planning expressed that such segregation would mean regression of the gains that have been done in terms of shared space and giving priority to non-motorized road users in the urban cityscape, and would be unfair to non-AV users. In the current non-expert group, we observe a dominant concern for safety overriding other issues related to equitable and fair sharing of space. The governance of the co-existence of AV with other road users is a primary ethical issue to be tackled.

Among drivers and RUD, there was also recognition of a negative impact of dedicating space to AV on the accessibility of other road users and non-AVs. Hence, although not expressed as such, fairness and social inclusion preoccupies the minds, more precisely minds of drivers and one RUD in the current sample.

The pedestrian group diverged from their initial position in favor of segregated space for safety and converged on equity by supporting the integration of the $\mathrm{AV}$ in public transport (some of them already tried several demo cases of automated shuttles) or car-sharing scheme, instead of private vehicles. This seemed to be an acceptable compromise to justify a dedicated lane for collective use rather than individual use and to improve equity. " $A n A V$ does not have priority over other road users. It should bring more equity [for all]."

In the RUD group, we observed a similar pattern of convergence on shared use, either by public transport or car-sharing, for the benefit of everyone. Additionally, RUD argued that their use of space is not always straightforward in order to maneuver the wheelchair. Moreover, they expressed the need to take into account the people in wheelchairs in the design of new shared spaces with AV to facilitate accessibility.

2.2.3 Scenario 3: Unavoidable accident. The interest of this scenario were the kind of sacrifice that participants were willing to accept and the ethics chart that they considered appropriate for AV. A general ethics chart for AV has not been retained during the discussions among participants. As in the previous scenarios, we grouped participants' responses in Table 4.

All groups had a consensus on giving priority to the protection of the pedestrian because, unlike the pedestrian, the driver is protected by the vehicle itself and by the passive safety systems. This is in line with the results of the famous Moral Machine project [e.g. 1]. Vulnerability of the victim at the target of the morally questionable act seems to determine the decision of permissibility.

One of the issues in the driver group was the possibility of letting the driver define the ethics settings of the vehicle as "they are the ones who have to live with the consequences". This is aligned with the view that AV becomes a moral proxy of its user, instead of a moral agent, and thus, should reflect the user's ethical preferences [10].

Two additional possibilities of risk mitigation and random decision were raised in the pedestrian group. The reason behind the risk mitigation argument was that "the situation is so complex that there will always be something that we will fail to take into account while programming. Hence, random [decision] would be the only fair way".

With respect to risk mitigation, the argument was that "we should take into account both. We do not settle saying beforehand that we absolutely protect one or the other".

\section{DISCUSSION}

The current article aims at reporting the results of three focus group sessions consisting of drivers, pedestrians, and road users with disabilities on the ethical issues raised by the deployment of AV conducted as part of the H2020 project SUaaVE.

The primary concern for an ethically aligned AV was responsibility among all road user groups. The large number of stakeholders involved in the design and deployment of automated transport blurs the lines of responsibility, leaving gaps for accountability, a 
Table 3: Opinions on the urban scenario across three groups

\begin{tabular}{lcc}
\hline & Drivers & Pedestrians \\
\hline Segregation of lanes for safety and efficiency purposes & 5 & 4 \\
Problem of exclusion \& accessibility for everyone & 2 & 3 \\
Problem of space for other road users when a lane is dedicated to AV & 2 & 2 \\
Not knowing the new rules of shared space and how to move & 1 & 1 \\
Prioritize AV in public transport to free space for vulnerable road users & 1 & \\
No need to change city design & - & 2 \\
\hline
\end{tabular}

Table 4: Opinions on the unavoidable accident scenario across three groups

\begin{tabular}{|c|c|c|c|}
\hline & Drivers & Pedestrians & RUD \\
\hline Brake to avoid hurting the pedestrian (and take over control) & 6 & 3 & 5 \\
\hline Mitigation by sharing the risk between the driver and the pedestrian & & 1 & 1 \\
\hline Random & & 1 & \\
\hline
\end{tabular}

phenomenon known as "problem of many hands" [9]. Interestingly, drivers were preoccupied also by the moral consequences of the AV actions, which is a new dimension to the usual emphasis on legal responsibility and accountability.

We observed clear distinctions among currently mobile and immobile road users in their reflections about human autonomy, wellbeing, and social justice. While all road user groups acknowledged the potential positive and negative impact of the AV on human autonomy and wellbeing, drivers were mainly concerned with loss of control and agency. RUD, to the contrary, reflected on the possibility of gaining autonomy and mobility. As RUD experience the challenges and injustice in the current transport system combined with economic precarity [11], they seem to be more sensitive to and knowledgeable about the implications new technologies for social justice and equity. This group was indeed the only one prioritizing social justice at Step 2. In fact, the issues related to human autonomy, wellbeing, and social justice become a question of accessibility, that is, access to basic rights and services for the road users currently limited in their mobility.

The similar points raised by different road user groups provide information to technology developers and policy makers about the potential bottlenecks for moral acceptability of AV technology. However, we also observe divergences among the different groups in their reasoning and prioritization of different principles. An inclusive approach to make sure that these diverging points are addressed in design and policy is of particular importance to ensure public acceptance.

The analysis of individual scenarios revealed interesting results. In the transgression of a traffic rule scenario, we observed a difference between the drivers and non-drivers. While drivers considered transgression permissible, non-drivers considered it impermissible. This may be related to the concerns about an anthropomorphic AV. The fact that a vehicle should remain as a machine and not be equipped with judgment faculties and feelings, like humans, has been raised on several occasions. In fact, the opacity of AIbased technologies and the transparency of the decision making process constitute a large item in several internationally recognized recommendations, such as European Commission's report on Trustworthy $\mathrm{AI}^{1}$.

The second scenario on the changes in the urban environment with the deployment of AV revealed a strong support for a separate lane for $\mathrm{AV}$ for the sakes of safety and efficiency. This result is counter-intuitive, given that the urban design is moving in the direction of shared space and co-existence of different road users. While the risks associated with the uncertainties about the AV may have led people to prefer a separate space for safety, counterarguments highlighted the unfairness of such exclusive use of space. Nonetheless, during the discussions we observed a distinction between the personal and collective use of AV, in favor of privileges for collective use to promote equity.

The last scenario on the unavoidable accident was also the most familiar one to the participants due to the large media coverage of the Moral Machine project. A clear pattern in favor of a Kantian principle of now harming an innocent [e.g. 12] emerged in all groups by the protection of the pedestrian. The vulnerability of the pedestrian seems to have contributed to participants' decision. In fact, perceived vulnerability underlies different moral emotions pertaining to protection and caring tendency [13]. Hence, it is possible that moral emotions mediate the permissibility of the action. Finally, an ethics policy based on claim mitigation has been studied by $[5,14]$. A study on the public acceptability of AV's decision making strategies showed that people considered a claim mitigation approach more fair and moral than absolute protection of one or the road user or a random decision process.

Overall, the ethical issues related to the deployment of AV were intertwined with public acceptability of this new technology among all road user groups in the current study. While the uncertainties about the technology underlie some worries about negative impacts, the potential benefits of the automated transport are also appreciated, provided the deployment addresses the needs and expectations of all road users.

${ }^{1}$ https://ec.europa.eu/digital-single-market/en/news/ethics-guidelines-trustworthyai 


\section{ACKNOWLEDGMENTS}

This research was conducted as part of the SUaaVE project "Supporting acceptance of automated vehicles". Grant sponsor: European Union's H2020 Research and Innovation Programme. Grant no: 814999.

\section{REFERENCES}

[1] Jean François Bonnefon, Azim Shariff, and Iyad Rahwan. 2016. The social dilemma of autonomous vehicles. Science, 352, 1573-1576. DOI: https://doi.org/10.1126/ science.aaf 2654

[2] Filippo Santoni de Sio. 2017. Killing by autonomous vehicles and the legal doctrine of necessity. Ethical Theory and Moral Practice, 20, 411-429. DOI: https://doi. org/10.1007/s10677-017-9780-7

[3] Geoff Keeling. 2018. Legal necessity, pareto efficiency \& justified killing in autonomous vehicle collisions. Ethical Theory and Moral Practice, 21,413 427.DOI:https://doi.org/10.1007/s10677-018-9887-5

[4] Derek Leben. 2017. A Rawlsian algorithm for autonomous vehicles. Ethics and Information Technologies, 19, 107-115. DOI: https://doi.org/10.1007/s10676-0179419-3

[5] Katherine Evans, Nelson de Moura, Stephane Chauvier, Raja Chatila, and Ebru Dogan. Ethical decision making in autonomous vehicles: the AVEthics project. Science and Engineering Ethics. https://doi.org/10.1007/s11948-020-00272-8

[6] Mladenovic, M. N., McPherson, T. 2016. Engineering social justice into traffic control for Self-Driving vehicles? Science and Engineering Ethics, 22, 1131-1149.
DOI: https://doi.org/10.1007/s11948-015-9690-9

[7] Sabine Roeser. 2006. The role of emotions in judging the moral acceptability of risks. Safety Science, 44, 689-700. DOI: https://doi.org/10.1016/j.ssci.2006.02.001

[8] Dimitris Milakis, Bart van Arem, and Bert van Wee, B. 2017. Policy and society related implications of automated driving: A review of literature and directions for future research. Journal of Intelligent Transportation Systems, 21, 324-348. DOI: https://doi.org/10.1080/15472450.2017.1291351

[9] Helen Nissenbaum. 1996. Accountability in a computerized society. Science and Engineering Ethics, 2, 25-42. DOI: https://doi.org/10.1007/BF02639315

[10] Sandberg, A., Bradshaw-Martin, H. 2013. What do Cars Think of Trolley Problems: Ethics for Autonomous Cars, in: Romportl, J., Ircing, P., Zackova, E., Polak, M., Schuster, R. (Eds.), Beyond AI: Artificial Golem Intelligence. Beyond AI 2013, University of West Bohemia, Pilsen, Czech Republic.

[11] Shane Epting. 2018. Automated vehicles and transportation justice. Philosophy \& Technology, 32, 389-403. DOI: https://doi.org/10.1007/s13347-018-0307-5

[12] Christophe Lutge, 2017. The German Ethics Code for Automated and Connected Driving. Philos. Technol. 30, 547-558. DOI: https://doi.org/10.1007/s13347-0170284-0

[13] Anton J.M. Dijker. 2010. Perceived vulnerability as a common basis of moral emotions. British Journal of Social Psychology, 49, 415-423. https://doi.org/10. 1348/014466609X482668

[14] Ebru Dogan, Katherine Evans, Nelson de Moura, Stephane Chauvier, and Raja Chatila. "A claim-mitigation approach to the ethical decisions of automated vehicles: a study on public acceptability". Submitted to Science and Engineering Ethics. 